\title{
Personal Note: Working with Farhad Arbab 1990-2005
}

\author{
Kees $\operatorname{Blom}^{(凶)}$ \\ CWI, P.O. Box 94079, 1090 GB Amsterdam, The Netherlands \\ Kees.Blom@cwi.nl
}

From 1990 to 2005 the author had the privilege and pleasure to work as a programmer in Farhad Arbab's research group, initially on the development, implementation and applications of the Manifold coordination language. During these years I learned to appreciate his broad scientific knowledge and technical insight, as well as his humor and warm personality.

The work on Manifold evolved in a number of moderate scale trials (a dozen or so cooperating computers) in different application fields $[1-4,7]$. The latter work on 'Chaotic Iteration' raised a question how to effectively implement Distributed Termination Detection (DTD) for sets of asynchronously connected processes without a central supervising process. This proved to be an old problem [5,8] for which many solutions had been proposed under various conditions [11], until recently [9]. Because at that time all existing DTD algorithms had serious drawback in terms of prerequisites and properties, a new experimental DTD algorithm was designed and implemented in Manifold. However, these experiments showed that other programming languages such as Java were more suitable for programming this type of algorithms.

This effort resulted in a Java implementation the BTTFWave protocol that was used in a number of applications, see [10] where also a short description of this protocol is given. Also, a formal correctness proof of the BTTFWave protocol has been presented in [12]. All these experiences may have helped to inspire Farhad in setting up a totally new paradigm for component composition: Reo [6], which in later years proved to be quite fruitful.

It so happened that around that time Farhad was appointed as a professor at Leiden University, and I was asked to join another research group. Although we managed by working in weekends and evening hours to complete specification, implementation and some applications of the BTTFWave protocol, Farhad never found the opportunity to publish more extensively on this work.

Hopefully this may change now that Farhad has reached the state of retirement, because in my opinion it is quite an elegant and effective protocol, that deserves a wider audience. In any event, I wish him all the best in years to come. 


\section{References}

1. Blom, C.L., Arbab, F., Hummel, S., Elshoff, I.J.P.: Coordination of a heterogeneous coastal hydrodynamics application in manifold. Technical report SEN-R9833, Centrum voor Wiskunde en Informatica, Kruislaan 413, 1098 SJ Amsterdam, The Netherlands, December 1998. https://ir.cwi.nl/pub/4589

2. Bonsanque, M.M., Arbab, F., de Bakker, J.W., Rutten, J.J.M.M., Scutella, A., Zavattaro, G.: A transition system semantics for the control-driven coordination language manifold. Theoret. Comput. Sci. 240(1), 3-47 (2000)

3. Everaars, C.T.H., Arbab, F., Koren, B.: Dynamic process composition and communication patterns in irregularly structured applications. Concurrency: Practice Exp. 12(2-3), 157-174 (2000)

4. Monfroy, E.: A coordination-based chaotic iteration algorithm for constraint propagation. In: Proceedings of the 2000 ACM Symposium on Applied Computing, SAC 2000, vol. 1, pp. 262-269. ACM, New York (2000)

5. Dijkstra, E.W.D., Scholten, C.S.: Termination detection for diffusing computations. Inf. Process. Lett. 11(1), 1-4 (1980)

6. Arbab, F.: Reo: a channel-based coordination model for component composition. Math. Struct. Comput. Sci. 14(3), 329-366 (2004)

7. Arbab, F., Blom, C.L., Burger, F.J., Everaars, C.T.H.: Reusable coordinator modules for massively concurrent applications. Softw.: Practice Exp. 28(7), 703-735 (1998). Extended version

8. Francez, N.: Distributed termination. ACM Trans. Program. Lang. Syst. 2(1), 42$55(1980)$

9. Árdal, K.B.: A fault-tolerant variant of the mahapatra-dutt termination detection algorithm. Master thesis, Vrije Universiteit, Amsterdam, 26 August 2017. http:// www.cs.vu.nl/ $\sim$ wanf/theses/ardal-mscthesis.pdf

10. Apt, K.R., Arbab, F., Ma, H.: A distributed platform for mechanism design. In: 2008 International Conference on Computational Intelligence for Modelling Control \& Automation, pp. 767-772. IEEE (2008)

11. Matocha, J., Camp, T.: A taxonomy of distributed termination detection algorithms. J. Syst. Softw. 43, 207-221 (1998)

12. Degenhardt, M.: Proving the correctness of the BTTF wave algorithm for distributed termination detection. Master thesis, Vrije Universiteit, Amsterdam, July 2014. http://www.cs.vu.nl/ $\sim$ wanf/theses/degenhardt.pdf 